\title{
Singapore Neonatal Resuscitation Guidelines 2021
}

\author{
Agnihotri Biswas ${ }^{1,2,3, *}$, MBBS, MRCPCH, Selina Kah Ying $\mathrm{Ho}^{2,4,5,6, *}$, MBBS, MMed, Wai Yan Yip ${ }^{2,5,6,7, *}$, MBBS, MRCPCH, \\ Khadijah Binti Abdul Kader ${ }^{1}$, MBBS, MRCPCH, Juin Yee Kong ${ }^{2,5,6,7}$, MD, FAAP, Kenny Teong Tai Ee $e^{3,8}$, MBBS, MRCP,

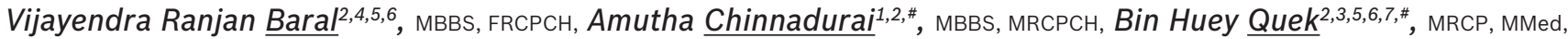 \\ Cheo Lian $\underline{Y e o}^{2,3,4,5,6, *}, \mathrm{MBBS}, \mathrm{MMed}$
}

\begin{abstract}
Neonatal resuscitation is a coordinated, team-based series of timed sequential steps that focuses on a transitional physiology to improve perinatal and neonatal outcomes. The practice of neonatal resuscitation has evolved over time and continues to be shaped by emerging evidence as well as key opinions. We present the revised Neonatal Resuscitation Guidelines for Singapore 2021. The recommendations from the International Liaison Committee on Resuscitation Neonatal Task Force Consensus on Science and Treatment Recommendations (2020) and guidelines from the American Heart Association and European Resuscitation Council were compared with existing guidelines. The recommendations of the Neonatal Subgroup of the Singapore Resuscitation and First Aid Council were derived after the work group discussed and appraised the current available evidence and their applicability to local clinical practice.
\end{abstract}

Keywords: neonatal resuscitation, newborn resuscitation, Singapore Resuscitation and First Aid Council (SRFAC) guidelines for resuscitation 2021

\section{BACKGROUND AND INTRODUCTION}

Resuscitation at birth requires a team of providers with different skill sets to communicate, collaborate and perform the necessary steps efficiently, guided by a standardised protocol and uniform practice guidelines. The majority of preterm newborns require some form of resuscitation and/or stabilisation during transition to extrauterine life. In comparison, about $10 \%$ of term newborns require stimulation to initiate breathing, $5 \%$ require positive pressure ventilation (PPV) before spontaneous breathing is established, $2 \%$ require intubation for longer respiratory support, $0.1 \%$ require cardiac compressions and $0.05 \%$ require adrenaline. ${ }^{(1-4)}$

This review by the Neonatal Subgroup of the Singapore Resuscitation and First Aid Council (SRFAC) is an update of the previous Singapore Neonatal Resuscitation Guidelines $(2016)^{(5)}$ and draws from the International Liaison Committee on Resuscitation (ILCOR) 2020 Consensus on Science with Treatment Recommendations (CoSTR), the American Heart Association guidelines for cardiopulmonary resuscitation and emergency cardiovascular care and the European Resuscitation Council newborn life support (2021) recommendations. ${ }^{(6-8)}$ This guideline applies to newborns transitioning to extrauterine life irrespective of their place of birth and neonates who have completed newborn transition and require resuscitation during the initial hospitalisation. The key highlights are presented in Box 1 and in the discussion on treatment recommendations and practice guidelines, which are organised in alignment with the newborn resuscitation algorithm (Fig. 1).

\section{ANTICIPATION AND PREPARATION FOR RESUSCITATION}

Identification of antepartum and intrapartum risk factors (Box 2) helps to anticipate the need for resuscitation at birth. Resuscitation may not be anticipated at all times; hence, it is important to be able to mobilise a team capable of advanced resuscitation at short notice. The topic on prediction of respiratory support in the delivery room was reviewed in 2020. ${ }^{(6)}$ There was no new evidence to support a change in the recommendations from the 2010 ILCOR guidelines. ${ }^{(9,10)}$

\section{Recommendations on anticipation and preparation}

- $\quad$ Anticipate resuscitation needs and prepare in advance.

- The environment should be warm, well-lit and draught-free, and should have a designated resuscitation area with an overhead warmer and equipment for monitoring.

- $\quad$ Equipment may be grouped by thermoregulation, ventilation and circulatory support, and kept well maintained and ready for use.

- Providers of newborn resuscitation should be trained in basic neonatal life support at the minimum.

- A team of advanced skilled providers trained in all steps of resuscitation should attend to high-risk deliveries.

\section{UMBILICAL CORD MANAGEMENT Delayed cord clamping}

Delayed cord clamping (DCC) by at least 30 seconds in term newborns increases haematocrit and reduces iron deficiency at the age of 3-6 months. ${ }^{(11)}$ However, there is no proven benefit in

\footnotetext{
${ }^{1}$ Department of Neonatology, Khoo Teck Puat-National University Children's Medical Institute, National University Health System, ${ }^{2}$ Department of Paediatrics, Yong Loo Lin School of Medicine, National University of Singapore, ${ }^{3}$ Neonatal Group, Paediatric Subcommittee, Singapore Resuscitation and First Aid Council, ${ }^{4}$ Department of Neonatal and Developmental Medicine, Singapore General Hospital, ${ }^{5}$ Duke-NUS Medical School, ${ }^{6}$ Lee Kong Chian School of Medicine, Nanyang Technological University, ${ }^{7}$ Department of Neonatology, KK Women's and Children's Hospital, ${ }^{8}$ Kinder Clinic Pte Ltd, Singapore

*These three authors contributed equally as first authors to this work. "These three authors contributed equally as senior authors to this work.

Correspondence: Dr Agnihotri Biswas, Senior Consultant, Department of Neonatology, Khoo Teck Puat-National University Children's Medical Institute, National University Health System, 1E Kent ridge Road, Level 12, Singapore 119228. biswas_agnihotri@nuhs.edu.sg
} 


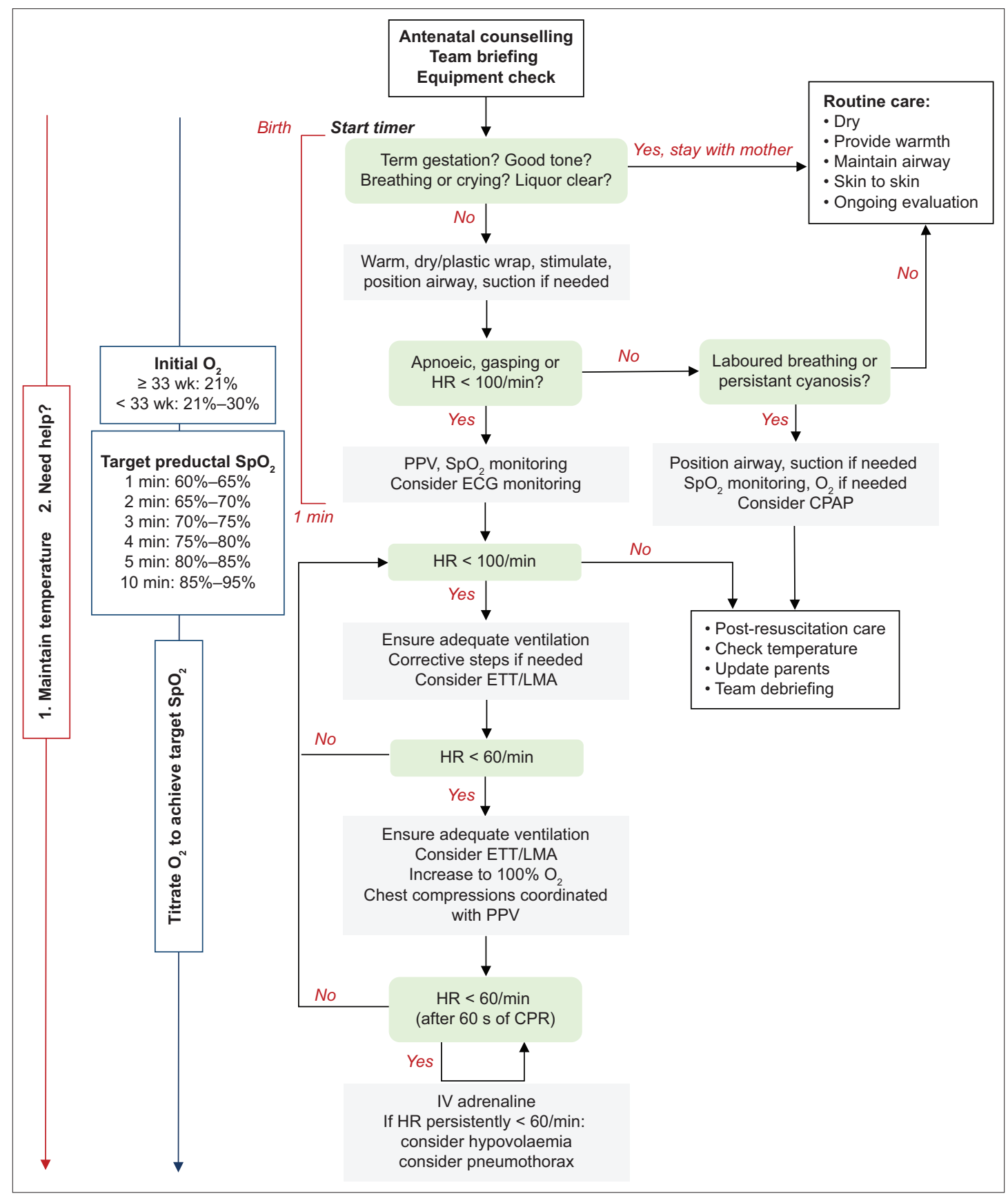

Fig. 1 Flowchart shows the newborn resuscitation algorithm. CPAP: continuous positive airway pressure; CPR: cardiopulmonary resuscitation; ECG: electrocardiography; ETT: endotracheal tube; HR: heart rate; IV: intravenous; LMA: laryngeal mask airway; $\mathrm{O}_{2}$ : oxygen; PPV: positive pressure ventilation; $\mathrm{SpO}_{2}$ : oxygen saturation

terms of reduction of mortality or longer-term neurodevelopmental outcome.

In vigorous preterm newborns, there is unequivocal evidence that DCC for 30-60 seconds reduces transfusions, necrotising enterocolitis (NEC) and all grades of intraventricular haemorrhage (IVH). ${ }^{(12-14)}$ However, evidence is unclear regarding the true benefit of DCC for preventing neurodevelopmental impairment in the early years, chronic lung disease (CLD), periventricular leukomalacia (PVL), late-onset sepsis and retinopathy of prematurity (ROP). ${ }^{(13-15)}$ For the critical outcome of in-hospital mortality after birth, accumulated evidence tends to favour DCC in babies born before 37 weeks. The relative risks of mortality in two large systematic reviews were $0.68,95 \%$ confidence interval $(\mathrm{Cl}) 0.52-0.90$ ( $\mathrm{n}=$
2,834), and $0.73,95 \% \mathrm{Cl} 0.54-0.98(\mathrm{n}=2,680) .{ }^{(13,14)}$ However, the benefits of DCC among 2,988 babies born at less than 34 weeks of gestation were equivocal, with a survival risk ratio (RR) of 1.02, 95\% Cl 1.00-1.04. ${ }^{(16)}$

\section{Umbilical cord milking}

Umbilical cord milking (UCM) has been performed with intact cord (i.e. intact-cord milking; ICM) or after cutting the cord (cut-cord milking). ICM has not been shown to provide survival advantage or lower morbidity over early cord clamping or DCC in preterms. ${ }^{(16)}$ Previous studies have shown increased risk of severe IVH with UCM among very premature newborns $\left(<28\right.$ weeks). ${ }^{(17,18)}$ 
Box 1. Key take-home messages for neonatal resuscitation:

1. Anticipation, preparation and having trained providers working as a team are essential for newborn resuscitation.

2. In newborns not requiring resuscitation at birth, delayed cord clamping for at least 30-60 seconds is recommended.

3. Maintain temperature of non-asphyxiated newborns between $36.5^{\circ} \mathrm{C}$ and $37.5^{\circ} \mathrm{C}$

4. In newborns requiring resuscitation, the 'golden minute' after birth is allocated for the provision of initial steps, re-evaluation and the initiation of positive pressure ventilation (PPV).

5. Perform ventilation corrective steps, if required, to achieve effective PPV, the most important step for successful newborn resuscitation.

6. An increase in heart rate (HR) is the most important indicator of effective resuscitation. Use auscultation for the initial assessment of HR and consider using electrocardiography for advanced resuscitation.

7. Commence PPV with $21 \%$ oxygen for newborns with gestational age $\geq 33$ weeks and $21 \%-30 \%$ oxygen for newborns with gestational age $<33$ weeks. Use pulse oximetry to titrate oxygen to preductal saturation targets.

8. Initiate chest compressions coordinated with PPV using $100 \%$ oxygen if the HR is $<60$ per minute after at least 30 seconds of effective PPV.

9. Administer adrenaline if $\mathrm{HR}$ is $<60$ per minute after at least 60 seconds of coordinated chest compressions and ventilation. Administer volume early in infants with suspected blood loss who are not responding to resuscitation.

10. Resuscitation may be discontinued if there is no HR after 20 minutes of optimum resuscitation and correction of reversible causes.

\section{Recommendations on cord management}

- DCC for 30-60 seconds is recommended for both stable term and preterm newborns who do not require resuscitation at birth.

- $\quad$ DCC takes precedence over cord blood banking.

- DCC should not delay resuscitation in babies who require immediate resuscitation.

- DCC is contraindicated in settings of monochorionic twins or when placental circulation is compromised (e.g. abruption, bleeding placenta praevia, cord avulsion).

- Cord milking is not recommended in preterm deliveries other than in research settings.

\section{THERMOREGULATION AND INITIAL STEPS \\ Thermoregulation}

A low admission temperature among non-asphyxiated term or preterm newborns is a strong predictor of mortality and morbidity. ${ }^{(19,20)}$ Hence, the earlier recommendation on recording an admission temperature is strengthened. . $5-8,21,22)$ Premature and very-low-birth-weight babies are at risk of hypothermia and require additional thermoregulatory interventions (e.g. overhead warmers, polyethylene wrap, exothermic mattress, warm humidified gas for ventilation). ${ }^{20,23-30)}$ For newborns who are
Box 2. Common risk factors that may predict the need for neonatal resuscitation:

Antepartum maternal factors

- High body mass index

- Past poor obstetric outcome

- Poorly controlled diabetes mellitus

- Pregnancy-induced hypertension

- Pre-existing chronic conditions

- Short stature

- Substance abuse

Antepartum fetal factors

- Fetal anaemia

- Genetic defects

- Growth restriction

- Intrauterine infection

- Major malformations

- Multifetal pregnancies

- Oligohydramnios

- Polyhydramnios

\section{Intrapartum factors}

- Abnormal cardiotocogram

- Antepartum haemorrhage

- Chorioamnionitis

- Cord prolapse

- Caesarean section under general anaesthesia

- Instrumental delivery

- Lack of antenatal steroids

- Prematurity or postmaturity

- Meconium-stained liquor

- Vaginal birth of a large baby or breech presentation

unintentionally hypothermic (temperature $<36^{\circ} \mathrm{C}$ ) on admission, current evidence does not support rapid $\left(\geq 0.5^{\circ} \mathrm{C} / \mathrm{hr}\right)$ over slow rewarming $\left(<0.5^{\circ} \mathrm{C} / \mathrm{hr}\right)^{\left({ }^{(31-34)}\right.}$ Their body temperature should be monitored during rewarming to avoid hyperthermia.

\section{Recommendations on temperature measurement and goals}

- Measure and record temperature after birth and upon admission.

- Skin, axillary or rectal temperature measurements are acceptable.

- Maintain temperature of non-asphyxiated newborns between $36.5^{\circ} \mathrm{C}$ and $37.5^{\circ} \mathrm{C}$.

- Prevent hypothermia $\left(<36^{\circ} \mathrm{C}\right)$ among non-asphyxiated newborns, and hyperthermia $\left(>38^{\circ} \mathrm{C}\right)$.

\section{Recommendations to prevent hypothermia}

- Maintain ambient environment of the delivery room at $23^{\circ} \mathrm{C}-25^{\circ} \mathrm{C}$.

- For preterm newborns at $<33$ weeks of gestation, an ambient temperature $\geq 25^{\circ} \mathrm{C}$ is preferred.

- Healthy term and preterm newborns with gestational age $\geq 33$ weeks who do not require resuscitation can be placed 
for skin-to-skin contact with the mother, both covered with a warm blanket for improvement of breastfeeding, temperature maintenance and glucose stability.

- $\quad$ Term and preterm babies with gestational age $\geq 33$ weeks who require resuscitative intervention should be placed under a radiant warmer and dried with warmed towels.

- $\quad$ Preterms with gestational age $<33$ weeks should be placed without drying in a polyethylene wrap or bag, under a radiant warmer. latrogenic hyperthermia should be avoided when multiple thermoregulatory interventions are simultaneously used.

- Thermoregulation should be maintained during the transfer of the newborn.

\section{Initial steps}

During the first 60 seconds of life, the 'golden minute', providers support the newborn transition to extrauterine life, and critical assessments are conducted to determine the need for resuscitation. ${ }^{(5-8,21,22)}$ Thermoregulatory steps mentioned previously are implemented during this phase. Most newborns, including preterms, initiate spontaneous breathing if they are adequately positioned with an open airway. ${ }^{(35)}$ Newborns with primary apnoea or irregular breathing may require gentle stimulation to attain regular breathing. Routine oropharyngeal or nasopharyngeal suctioning can be harmful, as it may precipitate apnoea and bradycardia, leading to hypoxaemia, longer time to normoxia, impaired cerebral blood flow and raised intracranial pressure. ${ }^{(36-44)}$

\section{Recommendations on initial steps}

- Ensure warmth.

- Gently rub the soles of the feet or back to stimulate breathing.

- Maintain an open airway by placing the newborn supine over a flat surface, with the neck in a neutral, sniffing position.

- $\quad$ Avoid routine suctioning. If the airway is obstructed, use a bulb sucker or suction catheter with a suction pressure of 80-100 mmHg to provide gentle and brief ( $<10$ seconds) suctioning.

- Assess the adequacy of breathing and stability of the heart rate $(\mathrm{HR})$ by the end of the golden minute to determine the need for further resuscitation.

\section{Management of babies born through meconium- stained liquor}

In utero passage of meconium is indicative of fetal stress and associated with fetal hypoxia-ischaemia. $5 \%-15 \%$ of all deliveries may be complicated by in utero passage of meconium, and up to $5 \%$ of babies born through meconium-stained liquor develop meconium aspiration syndrome (MAS). ${ }^{(45-47)}$ In 449 non-vigorous babies born through meconium-stained liquor, immediate laryngoscopy with or without tracheal suction showed no survival advantage compared with immediate resuscitation without laryngoscopy (RR 0.99, 95\% Cl 0.93-1.06). ${ }^{(47)}$ There was no difference in the outcomes of MAS, pulmonary hypertension, hypoxic ischaemic encephalopathy (HIE) or initial resuscitation requirements. Recent systematic reviews further support this finding. ${ }^{(48-52)}$

\section{Recommendations on management of babies born through meconium-stained liquor}

- Intrapartum suction of nose or mouth is contraindicated.

- In both vigorous and non-vigorous babies born through meconium-stained amniotic fluid, routine oropharyngeal or nasopharyngeal suctioning is not required. Bulb suctioning or suctioning using $\geq 10$-Fr size catheter may be required under direct vision if upper airways are suspected to be obstructed.

- $\quad$ Follow usual resuscitation steps in a non-vigorous baby to establish regular respiration. If PPV and ventilation correction steps do not result in lung inflation, the possibility of proximal lower airway obstruction by meconium may be considered, and tracheal suction may be attempted using an endotracheal tube (ETT) and meconium aspirator.

\section{ASSESSMENT OF THE NEWBORN}

During the initial steps, the provider needs to determine the transition needs of the newborn through assessment of breathing, tone and HR. Pallor, cyanosis, poor tone, weak cry in a term newborn, shallow or gasping breathing and bradycardia $(H R<100 / \mathrm{min})$ are important clues that indicate a need for further resuscitation.

\section{Assessment of heart rate}

An increase in the HR is the most important indicator of effective resuscitation. Auscultation remains the easiest method for the initial assessment of $\mathrm{HR}$, while pulse oximetry and three-lead electrocardiography (ECG) provide continuous and reliable HR assessment. ${ }^{(7)}$ ECG may be faster and more reliable than pulse oximetry or auscultation in providing accurate HR assessment, with no technical difficulties in applying the leads. ${ }^{(6,7,53)} \mathrm{A}$ study from a local neonatal unit showed that not only was it faster to apply the ECG leads than a pulse oximetry probe (27 seconds vs. 33.5 seconds, $p<0.001)$, but reliable data acquisition was also faster with ECG (10 seconds vs. 30.5 seconds, $p<0.001) .{ }^{(54)}$ However, ECG cannot replace the need for pulse oximetry to assess oxygenation and perfusion.

\section{Recommendations on assessment of heart rate and oxygen saturation}

- Auscultation is the preferred method for the initial assessment of HR. If a stethoscope is not available, umbilical cord palpation may be used.

- Use of ECG should be considered when advanced resuscitation is anticipated.

- Pulse oximetry should be used to evaluate oxygen saturation during resuscitation.

\section{POSITIVE PRESSURE VENTILATION Inspiratory and expiratory pressures for providing positive pressure ventilation}

Effective PPV is the most important step for successful resuscitation in newborns with apnoea or bradycardia. ${ }^{(22)}$ The majority of nonvigorous newborns respond to the initial steps and PPV, with the 
risk of death or prolonged admission increasing by $16 \%$ for every 30-second delay in initiating PPV. ${ }^{(7,55)}$

Inflation pressures up to $30 \mathrm{~cm} \mathrm{H}_{2} \mathrm{O}$ in term newborns and up to $25 \mathrm{~cm} \mathrm{H}_{2} \mathrm{O}$ in preterms are sufficient to inflate the lungs. High tidal volumes are associated with lung injury, impaired gas exchange and reduced lung compliance in immature animals, and severe IVH in preterm newborns..$^{(22,56)}$

During PPV, use of positive end-expiratory pressure (PEEP) helps to maintain alveolar recruitment, resulting in lower intubation rates, lower inspiratory pressure, shorter duration of PPV and higher number of infants resuscitated in room air. ${ }^{(57-60)}$ Use of PEEP is associated with increased survival to discharge without CLD, severe IVH or PVL (odds ratio 1.38, 95\% Cl 1.06-1.80). ${ }^{(61)}$

\section{Recommendations on pressure for providing PPV}

- $\quad$ PPV should be initiated within 60 seconds (the 'golden minute') after birth in newborns who remain apnoeic, gasping or bradycardic (HR $<100 /$ min) despite the initial steps.

- Initiate PPV with an inspiratory pressure of $20-25 \mathrm{~cm} \mathrm{H}_{2} \mathrm{O}$. Adjust inflation pressure to achieve optimal chest rise.

- In preterm infants with gestational age $<33$ weeks who require PPV, a PEEP of $5 \mathrm{~cm} \mathrm{H}_{2} \mathrm{O}$ is suggested. In newborns with gestational age $\geq 33$ weeks, provision of PEEP may be beneficial.

\section{Rate and inspiratory time during PPV}

A short inspiratory time of $<1$ second while providing PPV matches the natural breathing pattern in newborns. ${ }^{(62)}$ Sustained lung inflation (SLI) with an inspiratory time $>5$ seconds has shown a decreased requirement for mechanical ventilation compared with conventional PPV or continuous positive airway pressure (CPAP). ${ }^{(63-69)}$ However, a recent systematic review showed no difference in the primary outcome of death before discharge (RR 1.09, 95\% Cl 0.83-1.43) as well as other pre-specified critical outcomes. ${ }^{(70-72)}$ A large randomised controlled trial was terminated early owing to increased early death in newborns with gestational age $<29$ weeks who were randomised to SLI (RR 2.42, 95\% Cl 1.15-5.09). ${ }^{(73)}$

\section{Recommendations on rate and inspiratory time during PPV}

- In term and preterm newborns requiring PPV, it is reasonable to initiate PPV with an inspiratory time $\leq 1$ second, at a rate of 40-60 breaths per minute.

- In preterm newborns, we do not recommend routine initial SLI for $>5$ seconds to initiate resuscitation. SLI may be considered only in research settings.

\section{OXYGEN ADMINISTRATION}

Term and preterm newborns administered high oxygen concentrations are vulnerable to free radical formation and tissue injury. Oxygen use in newborns was reviewed by the NLS task force and published as a meta-analysis as well as an ILCOR CoSTR statement. ${ }^{(74,75)}$ Among newborns with a gestational age $\geq 35$ weeks who were receiving respiratory support at birth, 40 fewer deaths out of 1,000 deaths were observed with $21 \%$ vs. $100 \%$ oxygen, with no difference in HIE and long-term neurodevelopmental impairment. For preterm newborns with a gestational age $<35$ weeks, a comparison of lower $(\leq 50 \%$ ) vs. higher (>50\%) initial oxygen concentration showed no effect on short- or long-term morbidity or mortality.

The European Resuscitation Council (2021) has supported a recent European consensus statement recommending a gestational age-dependant graded use for the starting fraction of inspired oxygen $\left(\mathrm{FiO}_{2}\right.$ ). 30\% oxygen was recommended for newborns with gestational age $<28$ weeks, $21 \%-30 \%$ for 28-31 weeks and $21 \%$ for $>31$ weeks. . $^{(8,76)}$

\section{Recommendations on use of supplemental oxygen}

- For preterm newborns with gestational age $<33$ weeks, resuscitation should be initiated at $21 \%-30 \%$ oxygen. If an oxygen blender is unavailable, a self-inflating bag without a reservoir that is connected to pure oxygen delivers about $40 \%$ oxygen and may be an acceptable alternative.

- $\quad$ For newborns with gestational age $\geq 33$ weeks, resuscitation should be commenced at $21 \%$ oxygen.

- $\quad$ Supplemental oxygen should be titrated in accordance with target preductal (right hand) minute specific oxygen saturation ranges (Fig. 1).

\section{CONTINUOUS POSITIVE AIRWAY PRESSURE}

In spontaneously breathing preterm newborns with respiratory distress at birth, application of CPAP reduces the risk of death or bronchopulmonary dysplasia at 36 weeks (number needed to treat $=25$ ) compared with intubation or mechanical ventilation, with no differences in pneumothorax rates, IVH, NEC and ROP. ${ }^{(76-81)}$ The optimal PEEP during CPAP is unclear. A randomised controlled trial comparing higher $\left(6-8 \mathrm{~cm} \mathrm{H}_{2} \mathrm{O}\right)$ vs. lower (3-5 $\mathrm{cm} \mathrm{H}_{2} \mathrm{O}$ ) PEEP during neonatal resuscitation until the first 120 hours of life is currently under way. ${ }^{(82)}$

\section{Recommendation on the use of CPAP}

- $\quad$ Support spontaneously breathing preterm newborns in respiratory distress at birth with CPAP (PEEP of 5-6 $\mathrm{cm} \mathrm{H}_{2} \mathrm{O}$ ).

\section{ENDOTRACHEAL INTUBATION AND LARYNGEAL MASK AIRWAY \\ Endotracheal intubation}

Tracheal intubation may be considered at several time points during neonatal resuscitation. The size and depth of the ETT are calculated using several methods (Table I). ${ }^{(83-87)}$ The position of the ETT is confirmed by observing chest rise, auscultation for equal breath sounds and prompt increase in HR. Capnography or colorimetric detection of carbon dioxide may be used to ensure appropriate tracheal placement of the ETT, especially during the transport of an intubated newborn. ${ }^{(88-90)}$

\section{Recommendations for endotracheal intubation}

- Consider intubation with ETT if mask ventilation is ineffective, chest compressions are initiated, there is a 
Table I. Endotracheal tube (ETT) size and depth of tube placement for oral intubation.*

\begin{tabular}{|lllll|}
\hline $\begin{array}{l}\text { Gestational } \\
\text { age (wk) }\end{array}$ & $\begin{array}{l}\text { Estimated } \\
\text { weight }(\mathbf{g})\end{array}$ & $\begin{array}{l}\text { ETT size: } \\
\text { internal } \\
\text { diameter } \\
\text { (mm) }\end{array}$ & $\begin{array}{l}\text { Depth of } \\
\text { insertion } \\
\text { at upper } \\
\text { lip (cm) }\end{array}$ & $\begin{array}{l}\text { Suction } \\
\text { catheter } \\
\text { size (Fr) }\end{array}$ \\
\hline$<29^{\dagger}$ & $<1,000^{\dagger}$ & 2.5 & $5.5-6.5$ & 6 \\
\hline $29-34$ & $1,000-2,000$ & 3.0 & $7.0-8.0$ & 6 \\
\hline $35-38$ & $2,000-3,000$ & 3.5 & $8.0-9.0$ & 8 \\
\hline$>38$ & $3,000-4,000$ & 3.5 & $9.0-10.0$ & 8 \\
\hline
\end{tabular}

*The following formulae may be used to estimate the insertion depth of ETT at the upper lip: Tochen's formula: weight $(\mathrm{kg})+6 \mathrm{~cm}$ (e.g. for a weight of $1 / 2 / 3 \mathrm{~kg}$, the ETT depth is $7 / 8 / 9 \mathrm{~cm}$, respectively); nasal-tragus length (NTL) measured as the distance from the nasal septum to the tragus: NTL $(\mathrm{cm})+0.5 \mathrm{~cm}(<31$ weeks of gestation) and NTL $(\mathrm{cm})+1 \mathrm{~cm}$ ( $\geq 31$ weeks of gestation). †Tochen's formula may overestimate the depth of insertion in extremely preterm infants.

prolonged need for ventilation, there is requirement of tracheal toileting for presumed airway obstruction, and in special circumstances (e.g. diaphragmatic hernia).

- Use of capnography or colorimetric detection of carbon dioxide to confirm endotracheal (ET) intubation is suggested.

\section{Laryngeal mask airway}

A recent systematic review reported that respiratory management with laryngeal mask airway (LMA) is feasible for a defined subgroup of newborns (gestational age $\geq 34$ weeks, weight $>1,500 \mathrm{~g}$ ), although current evidence is insufficient to recommend the LMA instead of face mask ventilation in the delivery room. ${ }^{(91)}$

\section{Recommendation for laryngeal mask airway}

- $\quad$ LMA may be used as an airway device when intubation is not successful or feasible in late preterm and term newborns.

\section{CHEST COMPRESSION}

Based on expert opinion, newborns whose HR remains $<60 /$ min despite 30 seconds of effective ventilation require circulatory support to supply oxygenated blood to the brain and vital organs. Previous recommendations on neonatal cardiopulmonary resuscitation $(\mathrm{CPR})$ remain unchanged, ${ }^{(5-8,21,22)}$ with synchronised compression to ventilation at a ratio of $3: 1$. Animal studies that compared this with alternate ratios did not show any advantage with respect to return of circulation or survival. ${ }^{(92-96)}$ The twothumb-encircling hands technique of chest compression is associated with improved blood pressure and less provider fatigue compared with the two-finger technique. ${ }^{(97,98)}$ Chest compressions can be performed from the side or from above the head-end once the airway is secured, to facilitate placement of an umbilical venous (UV) catheter.

\section{Recommendations on chest compressions}

- $\quad$ Chest compressions should be commenced if the HR remains $<60 / \mathrm{min}$ after at least 30 seconds of effective PPV.

- When chest compressions are initiated, increase inspired oxygen to $100 \%$ and titrate to target, based on pulse oximetry.
- The two-thumb, hands-encircling-chest technique is recommended. Thumbs (overlapping thumb in a small newborn) are placed over the lower third of the sternum.

- Depress the sternum about one-third of the anteroposterior diameter of the chest and release to allow chest recoil and ventilation.

- Chest compressions should be coordinated with ventilation at a 3:1 ratio, providing 90 compressions and 30 inflations (120 events) per minute.

- $\quad$ Reassess HR, oxygen saturation and return of spontaneous breathing after every 60 seconds of coordinated chest compression and ventilation.

\section{MEDICATIONS Intravascular access}

In circumstances where circulation fails to return with synchronised PPV and chest compressions, medications and/or volume expanders are recommended to maintain circulation. UV catheterisation is a safe and easy vascular access. ${ }^{(7,99)}$ If this is not feasible or fails, medications and fluids may be delivered via the intraosseous $(\mathrm{IO})$ route. ${ }^{(22,99-101)}$ Case reports of tibial fractures, compartment syndrome from extravasation of fluid and medications, and amputation have been reported from IO access, particularly in preterm newborns..$^{100,102-106)}$

\section{Recommendations on intravascular access}

- UV catheterisation remains the standard method of vascular access in delivery room resuscitation.

- If UV access is not feasible, the IO route may be used with caution, especially in preterm newborns.

\section{Adrenaline}

Adrenaline, with its vasoconstrictive effect, increases blood flow to the coronary vessels, improving cardiac contractility and return of spontaneous circulation (ROSC). Studies comparing the efficacy of intravenous (IV) versus ET adrenaline in newborns demonstrate no significant difference in efficacy between initial ET and initial IV dose. ${ }^{(107,108)}$ However, most newborns require at least one dose of IV adrenaline before ROSC. ${ }^{(4,109)}$ An animal study showed faster and higher rates of ROSC with $0.3 \mathrm{~mL} / \mathrm{kg}$ of IV adrenaline compared with $1 \mathrm{~mL} / \mathrm{kg}$ of ET adrenaline. ${ }^{(110)}$ Use of saline flush following intravenous administration improves medication delivery. ${ }^{(111,112)}$

\section{Recommendations on use of adrenaline}

- If HR remains < 60/min after one minute of CPR, IV adrenaline ( 1 in 10,$000 ; 0.1 \mathrm{mg} / \mathrm{mL}$ ) is recommended at a dose of $0.1-0.3 \mathrm{~mL} / \mathrm{kg}(0.01-0.03 \mathrm{mg} / \mathrm{kg})$, administered rapidly followed by a $3-\mathrm{mL}$ saline flush.

- $\quad$ Continue CPR after adrenaline and assess response after 60 seconds. Repeat adrenaline every 3-5 minutes if HR remains $<60 / \mathrm{min}$.

- If the IV route is not immediately available, administer ET adrenaline ( 1 in 10,$000 ; 0.1 \mathrm{mg} / \mathrm{mL}$ ) at $0.5-1 \mathrm{~mL} / \mathrm{kg}$. Administer IV adrenaline once intravascular access is obtained. 


\section{Volume replacement}

Newborns who do not respond to advanced resuscitation may have hypovolaemia and occult blood loss; hence, a trial of volume administration may be considered..$^{(9,10)}$ Both animal and human studies support the use of crystalloids over albumin as the first choice for volume expansion. ${ }^{(113-117)}$

\section{Recommendations on volume replacement}

- Early volume replacement in newborns is recommended following non-response to advanced resuscitation, even if blood loss is not apparent.

- $10 \mathrm{~mL} / \mathrm{kg}$ of $0.9 \%$ saline administered over 5-10 minutes is the first choice.

- Uncross-matched O, Rh-negative blood is recommended when blood loss or anaemia is suspected.

\section{Use of sodium bicarbonate in resuscitation}

Severe metabolic acidosis during prolonged resuscitation may depress myocardial contractility. This forms the basis of considering sodium bicarbonate administration. There is, however, no role for its use during brief resuscitation..$^{(9,10)}$

\section{Recommendation on use of sodium bicarbonate}

- Consider sodium bicarbonate (2-4 mL/kg of $4.2 \%$ sodium bicarbonate) only in prolonged cardiac arrest unresponsive to adequate ventilation synchronised with chest compression, adrenaline and/or volume expansion.

\section{POST-RESUSCITATION CARE}

Newborns may continue to have substantial dysregulation of their metabolic and homeostatic adaptation following successful resuscitation. Hence, close monitoring and anticipatory care are required.

\section{Glucose control}

Newborns requiring resuscitation are prone to hypoglycaemia and impaired glucose homeostasis, which predispose them to adverse neurodevelopmental outcomes. ${ }^{(118-121)}$

\section{Recommendations on glucose control}

- Monitor blood glucose following resuscitation at appropriate intervals.

- Avoid hypoglycaemia, hyperglycaemia and wide fluctuations in blood glucose.

- Early institution of glucose infusion or parenteral nutrition is recommended for newborns at risk of hypoglycaemia.

\section{Therapeutic hypothermia}

Need for advanced resuscitation is highly predictive of HIE. ${ }^{(109,122-126)}$ A Cochrane review conclusively demonstrated that therapeutic hypothermia (TH) significantly reduces the risk of death or major neurodisability at 18 months in term and nearterm newborns with moderate to severe perinatal asphyxia (RR $0.75,95 \% \mathrm{Cl} 0.68-0.83) .{ }^{(127)}$ Current evidence is insufficient to recommend a change in practice for newborns with gestational age $<36$ weeks. There is insufficient evidence to recommend $\mathrm{TH}$ in the setting of mild HIE, delayed initiation for $>6$ hours, $\mathrm{TH}$ beyond 72 hours or target temperature below $33^{\circ} \mathrm{C} .{ }^{(128-130)}$

\section{Recommendations on therapeutic hypothermia}

- $\quad$ TH should be offered to infants with gestational age $\geq$ 36 weeks with moderate to severe HIE within six hours of life.

- $\quad$ TH should be performed as per established protocols, and core temperature should be maintained between $33^{\circ} \mathrm{C}$ and $34^{\circ} \mathrm{C}$ for 72 hours. Rewarming to $36.5^{\circ} \mathrm{C}$ should be achieved slowly in no less than four hours.

- Newborns requiring TH should be managed in centres that have the appropriate expertise and support, including the provision of longitudinal follow-up care.

\section{WITHHOLDING RESUSCITATION AND DISCONTINUING RESUSCITATIVE EFFORTS}

Options of withholding or discontinuing resuscitative efforts are best discussed with parents in the framework of shared decisionmaking. ${ }^{(131-135)}$ Both options are ethically equivalent. ${ }^{(136)}$

\section{Withholding resuscitation}

When a newborn faces certain early death or there is high anticipated burden in the event of survival owing to resuscitative measures, withholding resuscitation may be reasonable. A coordinated approach and communication between the parents and different stakeholders from the neonatal and obstetric teams ensure consistency in management. ${ }^{(137-139)}$ Maximal resuscitation should be provided until the parents have been consulted. ${ }^{(131)}$

\section{Recommendations on withholding resuscitation}

- $\quad$ Resuscitation of newborns with gestational age $<23$ weeks is not recommended.

- It may be reasonable to attempt resuscitation in newborns with gestational age ranging from $23^{+0}$ to $23^{+6}$ weeks if the parents express a strong wish for resuscitation. Decisions must be additionally guided by the certainty of gestation and other risk factors, condition at birth and response to initial resuscitation measures.

- At $24^{+0}$ to $24^{+6}$ weeks, it would be normal practice to offer full resuscitation unless the parents and clinicians make a shared decision against it based on the baby's clinical condition and best interest principles.

- When antenatally identified conditions are associated with almost certain early death or unacceptably poor outcomes among survivors (e.g. anencephaly), resuscitation should not be offered.

\section{Discontinuing resuscitation}

There is no evidence that any specific duration of resuscitation is universally predictive of mortality or adverse neurodevelopmental outcomes. ${ }^{(140)}$ Recent reports reveal a small but definite possibility of ROSC and acceptable outcome despite an Apgar score of 0 at ten minutes. ${ }^{(123,141)}$ Response to resuscitation may not be apparent 
until all steps of resuscitation are performed and reversible causes are treated; this may take longer than ten minutes. ${ }^{(142)}$

\section{Recommendations on discontinuing resuscitation}

- If 20 minutes of optimal resuscitation and correction of all reversible causes do not result in ROSC or cardiac activity, resuscitation may be discontinued.

- $\quad$ The decision to continue or discontinue resuscitative efforts must be individualised and discussed with the parents.

\section{OUT-OF-HOSPITAL RESUSCITATION OF NEWBORN INFANTS}

Emergency services dealing with unexpected births in the community should have trained personnel who are expected to carry the appropriate equipment. Out-of-hospital deliveries should ideally be attended by two personnel, one of whom should be competent in PPV and chest compression. ${ }^{\left({ }^{8}\right)}$

\section{Recommendations on out-of-hospital resuscitation of newborns}

- $\quad$ First responders are recommended to follow the neonatal resuscitation algorithm, with a focus on supporting transition, temperature maintenance and breathing with bag-mask ventilation, if required.

- $\quad$ ECG may be used to monitor the HR, or a portable pulse oximeter may be used to monitor HR and oxygen saturation at resuscitation and during transit.

- Information about gestation, number of babies, presence of recognised risk factors and need for resuscitation should be communicated early and clearly to the receiving hospital.

\section{BRIEFING AND DEBRIEFING}

Implementation of video-assisted, performance-focused debriefings improved neonatal resuscitation skills, adherence to best practice guidelines and team performance. ${ }^{(143)}$ The use of prebriefing and debriefing checklists during neonatal resuscitation was helpful in improving communication. ${ }^{(144,145)}$

\section{Recommendations on briefing and debriefing}

- $\quad$ Briefing before delivery should be conducted where possible.

- In a multi-member team, a team leader should assign roles and responsibilities to team members, anticipate interventions and provide a short briefing if time permits.

- Debriefing after neonatal resuscitation is encouraged.

\section{TRAINING FREQUENCY}

Several studies have shown that providers who received frequent refresher training and booster simulations showed better procedural skills and teamwork behaviours, with potential impact on neonatal outcomes. ${ }^{(146-148)}$

\section{Recommendation on retraining of providers}

- It is recommended that individual providers attend regular focused retraining of skills and knowledge at two-yearly intervals or more frequently, where possible.

\section{INFECTION PREVENTION AND CONTROL}

The 2003 severe acute respiratory syndrome outbreak and COVID-19 pandemic have led us to refocus our attention on ensuring appropriate infection prevention and control (IPC) measures during all neonatal resuscitation standbys. Specific recommendations relating to newborn resuscitation in COVID-19 for Singapore have recently been published. ${ }^{(149,150)}$ Additional institution-specific workflows pertaining to enhanced transmission-based precautions may be developed in collaboration with the individual IPC teams. Simulation training can facilitate safe neonatal resuscitation practices during specific IPC situations.

\section{Recommendations on IPC}

- $\quad$ Standard precautions should be observed for all neonatal deliveries.

- $\quad$ Transmission-based precautions are required in situations dealing with unique infections. Additional considerations on processes, preparation, site of delivery, personal protective equipment and personnel will ensure the safety of the patients and healthcare workers alike.

\section{CONCLUSION}

The 2020 guidelines from ILCOR, AHA and ERC 2021 are a testament to the extensive appraisal of information that has emerged since the 2015 review. While the 2015 resuscitation algorithm remains unchanged, many of the existing recommendations were reaffirmed. Some received stronger support (e.g. no routine tracheal suctioning of newborns delivered in the setting of meconium-stained liquor), while for others, caution was raised from emerging safety data (e.g. harm in preterm newborns following SLI). Still, many questions remain for future reviews (e.g. the optimal initial $\mathrm{FiO}_{2}$ for preterm newborns). The Singapore Neonatal Resuscitation Guidelines 2021 represent the consensus view of a workgroup of neonatologists who reviewed the current evidence and developed recommendations that are relevant for local practice.

\section{ACKNOWLEDGEMENTS}

The authors thank Prof Lim Swee Han, Chairman, SRFAC, and Senior Consultant, Department of Emergency Medicine, Singapore General Hospital; all the instructors of the Singapore Neonatal Resuscitation Course for their contributions in training and education; and Dr Dimple Rajgor for helping with the formatting and submission of this manuscript for publication.

\section{REFERENCES}

1. Ersdal HL, Linde J, Mduma E, Auestad B, Perlman J. Neonatal outcome following cord clamping after onset of spontaneous respiration. Pediatrics 2014; 134:265-72.

2. Niles DE, Cines $\mathrm{C}$, Insley $\mathrm{E}$, et al. Incidence and characteristics of positive pressure ventilation delivered to newborns in a US tertiary academic hospital. Resuscitation 2017; 115:102-9.

3. Perlman JM, Risser R. Cardiopulmonary resuscitation in the delivery room. Associated clinical events. Arch Pediatr Adolesc Med 1995; 149:20-5.

4. Halling C, Sparks JE, Christie L, Wyckoff MH. Efficacy of intravenous and endotracheal epinephrine during neonatal cardiopulmonary resuscitation in the delivery room. J Pediatr 2017; 185:232-6.

5. Yeo CL, Biswas A, Ee TT, et al. Singapore Neonatal Resuscitation Guidelines 
2016. Singapore Med J 2017; 58:391-403.

6. Wyckoff MH, Wyllie J, Aziz K, et al. Neonatal Life Support: 2020 international consensus on cardiopulmonary resuscitation and emergency cardiovascular care science with treatment recommendations. Circulation 2020; 142(16 Suppl 1):S185-221.

7. Aziz K, Lee HC, Escobedo MB, et al. Part 5: neonatal resuscitation: 2020 American Heart Association guidelines for cardiopulmonary resuscitation and emergency cardiovascular care. Circulation 2020; 142(16 Suppl 2):S524-50.

8. Madar J, Roehr CC, Ainsworth S, et al. European Resuscitation Council guidelines 2021: newborn resuscitation and support of transition of infants at birth. Resuscitation 2021; 161:291-326.

9. Perlman JM, Wyllie J, Kattwinkel J, et al. Part 11: neonatal resuscitation: 2010 international consensus on cardiopulmonary resuscitation and emergency cardiovascular care science with treatment recommendations. Circulation 2010; 122(16 Suppl 2):S516-38.

10. Wyllie J, Perlman JM, Kattwinkel J, et al. Part 11: neonatal resuscitation: 2010 international consensus on cardiopulmonary resuscitation and emergency cardiovascular care science with treatment recommendations. Resuscitation 2010; 81 Suppl 1:e260-87.

11. McDonald SJ, Middleton P, Dowswell T, Morris PS. Effect of timing of umbilical cord clamping of term infants on maternal and neonatal outcomes. Cochrane Database Syst Rev 2013; 2013:CD004074.

12. Rabe H, Diaz-Rossello JL, Duley L, Dowswell T. Effect of timing of umbilical cord clamping and other strategies to influence placental transfusion at preterm birth on maternal and infant outcomes. Cochrane Database Syst Rev 2012; (8):CD003248.

13. Fogarty M, Osborn DA, Askie L, et al. Delayed vs early umbilical cord clamping for preterm infants: a systematic review and meta-analysis. Am J Obstet Gynecol 2018; 218:1-18

14. Rabe H, Gyte GM, Diaz-Rossello JL, Duley L. Effect of timing of umbilical cord clamping and other strategies to influence placental transfusion at preterm birth on maternal and infant outcomes. Cochrane Database Syst Rev 2019; 9:CD003248

15. Tarnow-Mordi W, Morris J, Kirby A, et al. Delayed versus immediate cord clamping in preterm infants. N Engl J Med 2017; 377:2445-55.

16. Seidler AL, Gyte GML, Rabe H, et al. Umbilical cord management for newborns $<34$ weeks' gestation: a meta-analysis. Pediatrics 2021; 147:e20200576.

17. Balasubramanian H, Ananthan A, Jain V, Rao SC, Kabra N. Umbilical cord milking in preterm infants: a systematic review and meta-analysis. Arch Dis Child Fetal Neonatal Ed 2020; 105:572-80.

18. Katheria A, Reister F, Essers J, et al. Association of umbilical cord milking vs delayed umbilical cord clamping with death or severe intraventricular hemorrhage among preterm infants. JAMA 2019; 322:1877-86.

19. Javaudin F, Hamel V, Legrand A, et al. Unplanned out-of-hospital birth and risk factors of adverse perinatal outcome: findings from a prospective cohort. Scand J Trauma Resusc Emerg Med 2019; 27:26.

20. McCall EM, Alderdice F, Halliday HL, Vohra S, Johnston L. Interventions to prevent hypothermia at birth in preterm and/or low birth weight infants. Cochrane Database Syst Rev 2018; 2:CD004210.

21. Perlman JM, Wyllie J, Kattwinkel J, et al. Part 7: neonatal resuscitation: 2015 international consensus on cardiopulmonary resuscitation and emergency cardiovascular care science with treatment recommendations. Circulation 2015; 132(16 Suppl 1):S204-41.

22. Wyckoff MH, Aziz K, Escobedo MB, et al. Part 13: neonatal resuscitation: 2015 American Heart Association guidelines update for cardiopulmonary resuscitation and emergency cardiovascular care. Circulation 2015; 132(18 Suppl 2):S543-60.

23. Jia YS, Lin ZL, Lv H, et al. Effect of delivery room temperature on the admission temperature of premature infants: a randomized controlled trial. J Perinato 2013; 33:264-7

24. Almeida PG, Chandley J, Davis J, Harrigan RC. Use of the heated gel mattress and its impact on admission temperature of very low birth-weight infants. Adv Neonatal Care 2009; 9:34-9.

25. Singh A, Duckett J, Newton T, Watkinson M. Improving neonatal unit admission temperatures in preterm babies: exothermic mattresses, polythene bags or a traditional approach? J Perinatol 2010; 30:45-9.

26. McCarthy LK, O'Donnell CP. Warming preterm infants in the delivery room: polyethylene bags, exothermic mattresses or both? Acta Paediatr 2011; 100:1534-7.

27. Meyer MP, Owen LS, Te Pas AB. Use of heated humidified gases for early stabilization of preterm infants: a meta-analysis. Front Pediatr 2018; 6:319.

28. Laptook AR, Bell EF, Shankaran S, et al. Admission temperature and associated mortality and morbidity among moderately and extremely preterm infants. J Pediatr 2018; 192:53-9.e2.

29. Lyu Y, Shah PS, Ye XY, et al. Association between admission temperature and mortality and major morbidity in preterm infants born at fewer than 33 weeks' gestation. JAMA Pediatr 2015; 169:e150277.

30. Lunze K, Bloom DE, Jamison DT, Hamer DH. The global burden of neonata hypothermia: systematic review of a major challenge for newborn survival. BMC Med 2013; 11:24.
31. Motil KJ, Blackburn MG, Pleasure JR. The effects of four different radiant warmer temperature set-points used for rewarming neonates. J Pediatr 1974; 85:546-50.

32. Tafari N, Gentz J. Aspects of rewarming newborn infants with severe accidental hypothermia. Acta Paediatr Scand 1974; 63:595-600.

33. Racine J, Jarjoui E. Severe hypothermia in infants. Helv Paediatr Acta 1982; $37: 317-22$.

34. Sofer S, Yagupsky P, Hershkowits J, Bearman JE. Improved outcome of hypothermic infants. Pediatr Emerg Care 1986; 2:211-4.

35. Chua C, Schmölzer GM, Davis PG. Airway manoeuvres to achieve upper airway patency during mask ventilation in newborn infants - an historical perspective. Resuscitation 2012; 83:411-6.

36. Carrasco M, Martell M, Estol PC. Oronasopharyngeal suction at birth: effects on arterial oxygen saturation. J Pediatr 1997; 130:832-4.

37. Gungor S, Kurt E, Teksoz E, et al. Oronasopharyngeal suction versus no suction in normal and term infants delivered by elective cesarean section: a prospective randomized controlled trial. Gynecol Obstet Invest 2006; 61:9-14.

38. Gungor S, Teksoz E, Ceyhan T, et al. Oronasopharyngeal suction versus no suction in normal, term and vaginally born infants: a prospective randomised controlled trial. Aust N Z J Obstet Gynaecol 2005; 45:453-6.

39. Kohlhauser C, Bernert G, Hermon M, et al. Effects of endotracheal suctioning in high-frequency oscillatory and conventionally ventilated low birth weight neonates on cerebral hemodynamics observed by near infrared spectroscopy (NIRS). Pediatr Pulmonol 2000; 29:270-5.

40. Skov L, Ryding J, Pryds O, Greisen G. Changes in cerebral oxygenation and cerebral blood volume during endotracheal suctioning in ventilated neonates. Acta Paediatr 1992; 81:389-93.

41. Perlman JM, Volpe JJ. Suctioning in the preterm infant: effects on cerebral blood flow velocity, intracranial pressure, and arterial blood pressure. Pediatrics 1983; 72:329-34

42. van Bel F, van de Bor M, Baan J, Ruys JH. The influence of abnormal blood gases on cerebral blood flow velocity in the preterm newborn. Neuropediatrics 1988; 19:27-32.

43. Fisher DM, Frewen T, Swedlow DB. Increase in intracranial pressure during suctioning--stimulation vs. rise in PaCO2. Anesthesiology 1982; 57:416-7.

44. Konstantelos D, Ifflaender S, Dinger J, Rüdiger M. Suctioning habits in the delivery room and the influence on postnatal adaptation - a video analysis. J Perinat Med 2015; 43:777-82.

45. Wiswell TE. Delivery room management of the meconium-stained newborn. J Perinatol 2008; 28 Suppl 3:S19-26.

46. Rawat M, Nangia S, Chandrasekharan P, Lakshminrusimha S. Approach to infants born through meconium stained amniotic fluid: evolution based on evidence? Am J Perinatol 2018; 35:815-22.

47. Trevisanuto D, Strand ML, Kawakami MD, et al. Tracheal suctioning of meconium at birth for non-vigorous infants: a systematic review and metaanalysis. Resuscitation 2020; 149:117-26

48. Chettri S, Adhisivam B, Bhat BV. Endotracheal suction for nonvigorous neonates born through meconium stained amniotic fluid: a randomized controlled trial. J Pediatr 2015; 166:1208-13.e1.

49. Nangia S, Sunder S, Biswas R, Saili A. Endotracheal suction in term non vigorous meconium stained neonates-a pilot study. Resuscitation 2016; 105:79-84.

50. Singh SN, Saxena S, Bhriguvanshi A, et al. Effect of endotracheal suctioning just after birth in non-vigorous infants born through meconium stained amniotic fluid: a randomized controlled trial. Clin Epidemiology Glob Health 2019; 7:165-70.

51. Phattraprayoon N, Tangamornsuksan W, Ungtrakul T. Outcomes of endotracheal suctioning in non-vigorous neonates born through meconium-stained amniotic fluid: a systematic review and meta-analysis. Arch Dis Child Fetal Neonatal Ed 2021; 106:31-8.

52. Kumar A, Kumar P, Basu S. Endotracheal suctioning for prevention of meconium aspiration syndrome: a randomized controlled trial. Eur J Pediatr 2019; 178:1825-32.

53. Bjorland PA, Ersdal HL, Øymar K, Rettedal SI. Compliance with guidelines and efficacy of heart rate monitoring during newborn resuscitation: a prospective video study. Neonatology 2020; 117:175-81.

54. Tan MGS, Lee LW, Ereno I, et al. Heart rate determination using electrocardiogram in comparison with pulse oximeter at neonatal resuscitation: the Singapore General Hospital experience. Ann Pediatr Res 2020; 4:1047.

55. Ersdal HL, Mduma E, Svensen E, Perlman JM. Early initiation of basic resuscitation interventions including face mask ventilation may reduce birth asphyxia related mortality in low-income countries: a prospective descriptive observational study. Resuscitation 2012; 83:869-73.

56. Mian Q, Cheung PY, O'Reilly M, et al. Impact of delivered tidal volume on the occurrence of intraventricular haemorrhage in preterm infants during positive pressure ventilation in the delivery room. Arch Dis Child Fetal Neonatal Ed 2019; 104:F57-62.

57. Dawson JA, Schmölzer GM, Kamlin CO, et al. Oxygenation with T-piece versus self-inflating bag for ventilation of extremely preterm infants at birth: a randomized controlled trial. J Pediatr 2011; 158:912-8.e1-2.

58. Szyld E, Aguilar A, Musante GA, et al. Comparison of devices for newborn 
ventilation in the delivery room. J Pediatr 2014; 165:234-9.e3.

59. Thakur A, Saluja S, Modi M, et al. T-piece or self inflating bag for positive pressure ventilation during delivery room resuscitation: an RCT. Resuscitation 2015; 90:21-4.

60. Finer NN, Carlo WA, Duara S, et al. Delivery room continuous positive airway pressure/positive end-expiratory pressure in extremely low birth weight infants: a feasibility trial. Pediatrics 2004; 114:651-7.

61. Guinsburg R, de Almeida MFB, de Castro JS, et al. T-piece versus self-inflating bag ventilation in preterm neonates at birth. Arch Dis Child Fetal Neonatal Ed 2018; 103:F49-55.

62. te Pas AB, Wong C, Kamlin CO, et al. Breathing patterns in preterm and term infants immediately after birth. Pediatr Res 2009; 65:352-6.

63. Lindner W, Högel J, Pohlandt F. Sustained pressure-controlled inflation or intermittent mandatory ventilation in preterm infants in the delivery room? A randomized, controlled trial on initial respiratory support via nasopharyngeal tube. Acta Paediatr 2005; 94:303-9.

64. Lista G, Boni L, Scopesi F, et al. Sustained lung inflation at birth for preterm infants: a randomized clinical trial. Pediatrics 2015; 135:e457-64.

65. Lista G, Fontana P, Castoldi F, Cavigioli F, Dani C. Does sustained lung inflation at birth improve outcome of preterm infants at risk for respiratory distress syndrome? Neonatology 2011; 99:45-50.

66. van Vonderen JJ, Hooper SB, Hummler HD, Lopriore E, te Pas AB. Effects of a sustained inflation in preterm infants at birth. J Pediatr 2014; 165:903-8.e1.

67. Ngan AY, Cheung PY, Hudson-Mason A, et al. Using exhaled $\mathrm{CO}_{2}$ to guide initial respiratory support at birth: a randomised controlled trial. Arch Dis Child Fetal Neonatal Ed 2017; 102:F525-31.

68. El-Chimi MS, Awad HA, El-Gammasy TM, et al. Sustained versus intermittent lung inflation for resuscitation of preterm infants: a randomized controlled trial. J Matern Fetal Neonatal Med 2017; 30:1273-8.

69. Jiravisitkul P, Rattanasiri S, Nuntnarumit P. Randomised controlled trial of sustained lung inflation for resuscitation of preterm infants in the delivery room. Resuscitation $2017 ; 111: 68-73$.

70. Kapadia VS, Urlesberger B, Soraisham A, et al. Sustained lung inflations during neonatal resuscitation at birth: a meta-analysis. Pediatrics 2021; 147:e2020021204.

71. Foglia EE, Te Pas AB, Kirpalani $\mathrm{H}$, et al. Sustained inflation vs standard resuscitation for preterm infants: a systematic review and meta-analysis. JAMA Pediatr 2020; 174:e195897.

72. Bruschettini M, O'Donnell CP, Davis PG, et al. Sustained versus standard inflations during neonatal resuscitation to prevent mortality and improve respiratory outcomes. Cochrane Database Syst Rev 2020; 3:CD004953.

73. Kirpalani H, Ratcliffe SJ, Keszler M, et al. Effect of sustained inflations vs intermittent positive pressure ventilation on bronchopulmonary dysplasia or death among extremely preterm infants: the SAIL randomized clinical trial. JAMA 2019; 321:1165-75.

74. Welsford M, Nishiyama C, Shortt C, et al. Initial oxygen use for preterm newborn resuscitation: a systematic review with meta-analysis. Pediatrics 2019; 143:e20181828.

75. Soar J, Maconochie I, Wyckoff MH, et al. 2019 international consensus on cardiopulmonary resuscitation and emergency cardiovascular care science with treatment recommendations: summary from the Basic Life Support; Advanced Life Support; Pediatric Life Support; Neonatal Life Support; Education, Implementation, and Teams; and First Aid Task Forces. Circulation 2019; 140:e826-80.

76. Sweet DG, Carnielli V, Greisen G, et al. European consensus guidelines on the management of respiratory distress syndrome - 2019 update. Neonatology 2019; 115:432-50.

77. Schmölzer GM, Kumar M, Pichler G, et al. Non-invasive versus invasive respiratory support in preterm infants at birth: systematic review and meta-analysis. BMJ 2013; 347:f5980.

78. SUPPORT Study Group of the Eunice Kennedy Shriver NICHD Neonatal Research Network; Finer NN, Carlo WA, Walsh MC, et al. Early CPAP versus surfactant in extremely preterm infants. N Engl J Med 2010; 362:1970-9.

79. Sandri F, Plavka R, Ancora G, et al. Prophylactic or early selective surfactant combined with nCPAP in very preterm infants. Pediatrics 2010; 125:e1402-9.

80. Morley CJ, Davis PG, Doyle LW, et al. Nasal CPAP or intubation at birth for very preterm infants. N Engl J Med 2008; 358:700-8.

81. Dunn MS, Kaempf J, de Klerk A, et al. Randomized trial comparing 3 approaches to the initial respiratory management of preterm neonates. Pediatrics 2011; 128:e1069-76.

82. Waitz M, Engel C, Schloesser R, et al. Application of two different nasal CPAP levels for the treatment of respiratory distress syndrome in preterm infants- "The OPTTIMMAL-Trial"-Optimizing PEEP To The IMMAture Lungs: study protocol of a randomized controlled trial. Trials 2020; 21:822.

83. Leung C. Optimal insertion depth for endotracheal tubes in extremely low-birthweight infants. Pediatr Crit Care Med 2018; 19:328-31.

84. Takeuchi S, Arai J, Nagafuji M, et al. Ideal endotracheal tube insertion depth in neonates with a birthweight less than 750 g. Pediatr Int 2020; 62:932-6.

85. Bartle RM, Miller AG, Diez AJ, et al. Evaluating endotracheal tube depth in infants weighing less than 1 kilogram. Respir Care 2019; 64:243-7.

86. Uygur Ö, Öncel MY, Şimşek GK, et al. Is nasal septum-tragus length measurement appropriate for endotracheal tube intubation depth in neonates? A randomized controlled study. Am J Perinatol 2021; 38:728-33.

87. Peterson J, Johnson N, Deakins K, et al. Accuracy of the 7-8-9 rule for endotracheal tube placement in the neonate. J Perinatol 2006; 26:333-6.

88. Repetto JE, Donohue PA-C PK, Baker SF, Kelly L, Nogee LM. Use of capnography in the delivery room for assessment of endotracheal tube placement. J Perinatol 2001; 21:284-7.

89. Roberts WA, Maniscalco WM, Cohen AR, Litman RS, Chhibber A. The use of capnography for recognition of esophageal intubation in the neonatal intensive care unit. Pediatr Pulmonol 1995; 19:262-8.

90. Aziz HF, Martin JB, Moore JJ. The pediatric disposable end-tidal carbon dioxide detector role in endotracheal intubation in newborns. J Perinatol 1999; 19:110-3.

91. Bansal SC, Caoci S, Dempsey E, Trevisanuto D, Roehr CC. The laryngeal mask airway and its use in neonatal resuscitation: a critical review of where we are in 2017/2018. Neonatology 2018; 113:152-61.

92. Solevåg AL, Schmölzer GM, O'Reilly M, et al. Myocardial perfusion and oxidative stress after $21 \%$ vs. $100 \%$ oxygen ventilation and uninterrupted chest compressions in severely asphyxiated piglets. Resuscitation 2016; 106:7-13.

93. Schmölzer GM, O'Reilly M, Labossiere J, et al. 3:1 compression to ventilation ratio versus continuous chest compression with asynchronous ventilation in a porcine model of neonatal resuscitation. Resuscitation 2014; 85:270-5.

94. Solevåg AL, Dannevig I, Wyckoff M, Saugstad OD, Nakstad B. Extended series of cardiac compressions during CPR in a swine model of perinatal asphyxia. Resuscitation 2010; 81:1571-6.

95. Solevåg AL, Dannevig I, Wyckoff M, Saugstad OD, Nakstad B. Return of spontaneous circulation with a compression: ventilation ratio of 15:2 versus $3: 1$ in newborn pigs with cardiac arrest due to asphyxia. Arch Dis Child Fetal Neonatal Ed 2011; 96:F417-21.

96. Pasquin MP, Cheung PY, Patel S, et al. Comparison of different compression to ventilation ratios $(2: 1,3: 1$, and $4: 1)$ during cardiopulmonary resuscitation in a porcine model of neonatal asphyxia. Neonatology 2018; 114:37-45.

97. David R. Closed chest cardiac massage in the newborn infant. Pediatrics 1988; 81:552-4.

98. Christman C, Hemway RJ, Wyckoff MH, Perlman JM. The two-thumb is superior to the two-finger method for administering chest compressions in a manikin model of neonatal resuscitation. Arch Dis Child Fetal Neonatal Ed 2011; 96:F99-101.

99. Kattwinkel J, Perlman JM, Aziz K, et al. Part 15: neonatal resuscitation: 2010 American Heart Association guidelines for cardiopulmonary resuscitation and emergency cardiovascular care. Circulation 2010; 122(18 Suppl 3):S909-19.

100. Ellemunter $H$, Simma B, Trawöger $R$, Maurer $H$. Intraosseous lines in preterm and full term neonates. Arch Dis Child Fetal Neonatal Ed 1999; 80:F74-5.

101. Wagner M, Olischar M, O'Reilly M, et al. Review of routes to administer medication during prolonged neonatal resuscitation. Pediatr Crit Care Med 2018; 19:332-8.

102. Vidal R, Kissoon N, Gayle M. Compartment syndrome following intraosseous infusion. Pediatrics 1993; 91:1201-2.

103. Katz DS, Wojtowycz AR. Tibial fracture: a complication of intraosseous infusion. Am J Emerg Med 1994; 12:258-9.

104. Carreras-González E, Brió-Sanagustín S, Guimerá I, Crespo C. [Complication of the intraosseous route in a newborn infant]. Med Intensiva 2012; 36:233-4. Spanish.

105. Oesterlie GE, Petersen KK, Knudsen L, Henriksen TB. Crural amputation of a newborn as a consequence of intraosseous needle insertion and calcium infusion. Pediatr Emerg Care 2014; 30:413-4.

106. Suominen PK, Nurmi E, Lauerma K. Intraosseous access in neonates and infants: risk of severe complications - a case report. Acta Anaesthesiol Scand 2015; 59:1389-93.

107. Hudak ML. Epinephrine for neonatal resuscitation: the limits of knowledge. Pediatrics 2020; 146:e2020019968.

108. Isayama T, Mildenhall L, Schmölzer GM, et al. The route, dose, and interval of epinephrine for neonatal resuscitation: a systematic review. Pediatrics 2020; 146:e20200586.

109. Barber CA, Wyckoff MH. Use and efficacy of endotracheal versus intravenous epinephrine during neonatal cardiopulmonary resuscitation in the delivery room. Pediatrics 2006; 118:1028-34.

110. Vali $P$, Chandrasekharan $P$, Rawat $M$, et al. Evaluation of timing and route of epinephrine in a neonatal model of asphyxial arrest. J Am Heart Assoc 2017; 6:e004402.

111. Vali P, Sankaran D, Rawat M, Berkelhamer S, Lakshminrusimha S. Epinephrine in neonatal resuscitation. Children (Basel) 2019; 6:51.

112. Sankaran D, Chandrasekharan PK, Gugino SF, et al. Randomised trial of epinephrine dose and flush volume in term newborn lambs. Arch Dis Child Fetal Neonatal Ed 2021 Mar 9. https://doi.org/10.1136/archdischild-2020-321034. [Epub ahead of print]

113. Niermeyer S. Volume resuscitation: crystalloid versus colloid. Clin Perinatol 2006; 33:133-40, viii.

114. Shalish W, Olivier F, Aly H, Sant'Anna G. Uses and misuses of albumin during resuscitation and in the neonatal intensive care unit. Semin Fetal Neonatal Med 2017; 22:328-35. 
115. Keir AK, Karam O, Hodyl N, et al. International, multicentre, observational study of fluid bolus therapy in neonates. J Paediatr Child Health 2019; 55:632-9.

116. Wyckoff M, Garcia D, Margraf L, Perlman J, Laptook A. Randomized trial of volume infusion during resuscitation of asphyxiated neonatal piglets. Pediatr Res 2007; 61:415-20.

117. Mendler MR, Schwarz S, Hechenrieder L, et al. Successful resuscitation in a model of asphyxia and hemorrhage to test different volume resuscitation strategies. A study in newborn piglets after transition. Front Pediatr 2018; 6:192.

118. Azzopardi D, Strohm B, Linsell L, et al. Implementation and conduct of therapeutic hypothermia for perinatal asphyxial encephalopathy in the UK-analysis of national data. PLoS One 2012; 7:e38504.

119. Basu SK, Ottolini K, Govindan V, et al. Early glycemic profile is associated with brain injury patterns on magnetic resonance imaging in hypoxic ischemic encephalopathy. J Pediatr 2018; 203:137-43.

120. Salhab WA, Wyckoff MH, Laptook AR, Perlman JM. Initial hypoglycemia and neonatal brain injury in term infants with severe fetal acidemia. Pediatrics $2004 ; 114: 361-6$

121. Nadeem M, Murray DM, Boylan GB, Dempsey EM, Ryan CA. Early blood glucose profile and neurodevelopmental outcome at two years in neonatal hypoxic-ischaemic encephalopathy. BMC Pediatr 2011; 11:10.

122. Laptook AR, Shankaran S, Ambalavanan N, et al. Outcome of term infants using apgar scores at 10 minutes following hypoxic-ischemic encephalopathy. Pediatrics 2009; 124:1619-26.

123. Ayrapetyan M, Talekar K, Schwabenbauer K, et al. Apgar scores at 10 minutes and outcomes in term and late preterm neonates with hypoxic-ischemic encephalopathy in the cooling era. Am J Perinatol 2019; 36:545-54.

124. Kasdorf E, Laptook A, Azzopardi D, Jacobs S, Perlman JM. Improving infant outcome with a 10 min Apgar of 0. Arch Dis Child Fetal Neonatal Ed 2015; 100:F102-5.

125. Harrington DJ, Redman CW, Moulden M, Greenwood CE. The long-term outcome in surviving infants with Apgar zero at 10 minutes: a systematic review of the literature and hospital-based cohort. Am J Obstet Gynecol 2007; 196:463.e1-5.

126. Wyckoff MH, Salhab WA, Heyne RJ, et al. Outcome of extremely low birth weight infants who received delivery room cardiopulmonary resuscitation. J Pediatr 2012; 160:239-44.e2.

127. Jacobs SE, Berg M, Hunt R, et al. Cooling for newborns with hypoxic ischaemic encephalopathy. Cochrane Database Syst Rev 2013; 2013:CD003311.

128. Shankaran S, Laptook AR, Pappas A, et al. Effect of depth and duration of cooling on deaths in the NICU among neonates with hypoxic ischemic encephalopathy: a randomized clinical trial. JAMA 2014; 312:2629-39.

129. Laptook AR, Shankaran S, Tyson JE, et al. Effect of therapeutic hypothermia initiated after 6 hours of age on death or disability among newborns with hypoxic-ischemic encephalopathy: a randomized clinical trial. JAMA 2017; 318:1550-60

130. Kariholu U, Montaldo P, Markati T, et al. Therapeutic hypothermia for mild neonatal encephalopathy: a systematic review and meta-analysis. Arch Dis Child Fetal Neonatal Ed 2020; 105:225-8

131. Lemyre B, Moore G. Counselling and management for anticipated extremely preterm birth. Paediatr Child Health 2017; 22:334-41.

132. Patel RM, Rysavy MA, Bell EF, Tyson JE. Survival of infants born at periviable gestational ages. Clin Perinatol 2017; 44:287-303.

133. Myrhaug HT, Brurberg KG, Hov L, Markestad T. Survival and impairment of extremely premature infants: a meta-analysis. Pediatrics 2019; 143:e20180933.

134. Lien Centre for Palliative Care, Duke-NUS Medical School, Singapore. LCPC guidance on supportive and comfort care in vulnerable babies 2018. Available at: https://www.duke-nus.edu.sg/docs/librariesprovider12/neonatal-guidance/ guidance-on-supportive-and-comfort-care-in-vulnerable-babies-2018. pdf?sfvrsn=85c94b1d_4. Accessed May 14, 2021.

135. Guillén U, Weiss EM, Munson D, et al. Guidelines for the management of extremely premature deliveries: a systematic review. Pediatrics 2015; 136:343-50.

136. Wilkinson D, Butcherine E, Savulescu J. Withdrawal aversion and the equivalence test. Am J Bioeth 2019; 19:21-8.

137. Larcher V, Craig F, Bhogal K, et al. Making decisions to limit treatment in lifelimiting and life-threatening conditions in children: a framework for practice. Arch Dis Child 2015; 100 Suppl 2:S3-23.

138. Kukora SK, Boss RD. Values-based shared decision-making in the antenatal period. Semin Fetal Neonatal Med 2018; 23:17-24

139. Mactier H, Bates SE, Johnston T, et al. Perinatal management of extreme preterm birth before 27 weeks of gestation: a framework for practice. Arch Dis Child Fetal Neonatal Ed 2020; 105:232-9.

140. Foglia EE, Weiner G, de Almeida MFB, et al. Duration of resuscitation at birth, mortality, and neurodevelopment: a systematic review. Pediatrics 2020; 146:e20201449.

141. Natarajan G, Shankaran S, Laptook AR, et al. Apgar scores at $10 \mathrm{~min}$ and outcomes at 6-7 years following hypoxic-ischaemic encephalopathy. Arch Dis Child Fetal Neonatal Ed 2013; 98:F473-9.

142. Zhong YJ, Claveau M, Yoon EW, et al. Neonates with a 10-min Apgar score of zero: outcomes by gestational age. Resuscitation 2019; 143:77-84.

143. Skare C, Calisch TE, Saeter E, et al. Implementation and effectiveness of a videobased debriefing programme for neonatal resuscitation. Acta Anaesthesiol Scand 2018; 62:394-403.

144. Sauer CW, Boutin MA, Fatayerji AN, et al. Delivery room quality improvement project improved compliance with best practices for a community NICU. Sci Rep 2016; 6:37397.

145. Katheria A, Rich W, Finer N. Development of a strategic process using checklists to facilitate team preparation and improve communication during neonatal resuscitation. Resuscitation 2013; 84:1552-7.

146. Tabangin ME, Josyula S, Taylor KK, Vasquez JC, Kamath-Rayne BD. Resuscitation skills after Helping Babies Breathe training: a comparison of varying practice frequency and impact on retention of skills in different types of providers. Int Health 2018; 10:163-71.

147. Bender J, Kennally K, Shields R, Overly F. Does simulation booster impact retention of resuscitation procedural skills and teamwork? J Perinatol 2014; 34:664-8.

148. Mduma $\mathrm{E}$, Ersdal $\mathrm{H}$, Svensen $\mathrm{E}$, et al. Frequent brief on-site simulation training and reduction in 24-h neonatal mortality--an educational intervention study. Resuscitation 2015; 93:1-7.

149. Quek BH, Biswas A, Ee KT, Yeo CL. Newborn resuscitation in COVID-19. Ann Acad Med Singap 2020; 49:909-12.

150. Yeo KT, Biswas A, Ho SKY, et al. Guidance for the clinical management of infants born to mothers with suspected/confirmed COVID-19 in Singapore. Singapore Med J 2021 Apr 19. https://doi.org/10.11622/smedj.2021045. [Epub ahead of print] 